\title{
BMJ Open Serum soluble urokinase type plasminogen activated receptor and focal segmental glomerulosclerosis: a systematic review and meta-analysis
}

\author{
Tiankui Shuai, ${ }^{1,2,3}$ Yan Pei Jing, ${ }^{2,4}$ Qiangru Huang, ${ }^{1,2,3}$ Huaiyu Xiong, ${ }^{1,2,3}$ \\ Jingjing Liu, ${ }^{1,2,3}$ Lei Zhu, ${ }^{1,2,3}$ Kehu Yang, ${ }^{2,4,5,6,7}$ Liu Jian (1) ${ }^{1,3}$
}

To cite: Shuai T, Pei Jing Y, Huang $Q$, et al. Serum soluble urokinase type plasminogen activated receptor and focal segmental glomerulosclerosis: a systematic review and meta-analysis. BMJ Open 2019;9:e031812. doi:10.1136/ bmjopen-2019-031812

- Prepublication history and additional material for this paper are available online. To view these files, please visit the journal online (http://dx.doi org/10.1136/bmjopen-2019031812).

TS and YPJ contributed equally.

Received 31 May 2019

Revised 04 September 2019

Accepted 10 September 2019

Check for updates

(C) Author(s) (or their employer(s)) 2019. Re-use permitted under CC BY-NC. No commercial re-use. See rights and permissions. Published by BMJ.

For numbered affiliations see end of article.

Correspondence to

Professor Liu Jian;

medecinliu@sina.com

Professor Kehu Yang; kehuyangebm2006@126.com

\section{ABSTRACT}

Objectives Soluble urokinase plasminogen activated receptor (suPAR) is a biomarker that may predict the occurrence of focal segmental glomerulosclerosis (FSGS); however, there is still controversy about whether suPAR can predict FSGS. In this study, we performed a systematic evaluation and meta-analysis to prove whether suPAR can predict FSGS, and to detect a threshold concentration of suPAR that can be used to diagnose FSGS. In addition, a threshold concentration of suPAR for the diagnosis of FSGS was proposed.

Design Systematic review and meta-analysis.

Data sources We systematically searched PubMed, Embase, Cochrane Library, Web of Science and China Biology Medicine databases for studies published from the inception dates to 1 December 2018.

Eligibility criteria (1) Data involving the suPAR level were from blood samples; (2) FSGS was diagnosed by biopsy; and (3) randomised controlled trials, cohort studies, casecontrol studies and cross-sectional studies.

Data extraction and synthesis Initially, a total of 364 studies were searched, among which 29 studies were finally included. In addition, seven studies described the cut-off value of suPAR, which ranged from 2992.6 to $5500 \mathrm{pg} / \mathrm{mL}$

Results The results showed that the suPAR levels in the primary FSGS group were significantly higher when compared with that in the normal control group $(p<0.001$; standard mean difference (SMD): $2.56 ; 95 \% \mathrm{Cl} 1.85$ to 3.28), and significant differences were observed in the secondary FSGS and in the normal control group $(p<0.001$ SMD: $1.68 ; 95 \% \mathrm{Cl} 1.37$ to 1.98 ). A suPAR concentration of $3000 \mathrm{pg} / \mathrm{mL}$ may be the best threshold for the diagnosis of primary FSGS (sensitivity $=0.72$; specificity $=0.88$; area under the curve $=0.85$ ).

Conclusion Our results suggested that suPAR might be a potential biomarker for predicting primary and secondary FSGS. In addition, our data showed that a suPAR concentration of $3000 \mathrm{pg} / \mathrm{mL}$ might be used as a threshold for the diagnosis of FSGS.

Trial registration number CRD42019120948.

\section{INTRODUCTION}

Focal segmental glomerulosclerosis (FSGS) is a pathological condition, and clinical
Strengths and limitations of this study

- In this study, we evaluated for the first time the threshold of the soluble urokinase plasminogen activated receptor level in the diagnosis of primary focal segmental glomerulosclerosis.

- We present evidence to distinguish different types of idiopathic nephrotic syndrome.

- Our study included both interventional and diagnostic meta-analyses.

- Heterogeneity has been explored; however, the source of heterogeneity has not yet been identified.

- The sample size of some of the included studies is small.

manifestations can include proteinuria and nephrotic syndrome. ${ }^{1}$ The mechanism of FSGS involves podocyte injury, which can result in degeneration of all nephrons and ultimately lead to chronic kidney disease (CKD). ${ }^{1}$ CKD is a global public health problem with a global prevalence of $11 \%-13 \%$ and is increasing rapidly. ${ }^{23}$ Moreover, in a recent study, it was demonstrated that the annual incidence rate of FSGS ranged from 0.2 to $1.8 / 100000$ per year. ${ }^{4}$ In general, there is no clinical manifestation in the early stage of FSGS, which often delays diagnosis and increases mortality. ${ }^{5}$ At present, diagnostic markers of kidney diseases are limited; however, several markers related to podocyte injury may play an important role in predicting disease progression.

Soluble urokinase plasminogen activated receptor (suPAR), a marker of podocyte injury, has been implicated in the pathogenesis of various kidney diseases. ${ }^{6}$ In a recent study, it was suggested that suPAR might be a biomarker for the diagnosis of kidney disease. ${ }^{7}$ In addition, in several studies, the relationship between suPAR and FSGS was explored; however, the results were controversial. $^{8-11}$ High-quality meta-analysis has 
been increasingly regarded a key tool for achieving evidence. ${ }^{12}{ }^{13}$ In a previous meta-analysis, ${ }^{14}$ it was shown that the concentration of suPAR was higher in patients with FSGS when compared with normal subjects; however, the heterogeneity was greater, and due to the small number of included studies no subgroup analysis was performed. Our meta-analysis included higher number of studies, a subgroup analysis, and sensitivity and specificity analyses for the diagnosis of the FSGS threshold using the concentration of suPAR. Furthermore, we also analysed whether the concentration of suPAR could be used to differentiate FSGS, minimal change disease (MCD) and membranous nephropathy (MN). Thus, this meta-analysis was conducted to explore whether suPAR could diagnose FSGS and to identify reasonable cut-offs of suPAR.

\section{METHODS}

This meta-analysis was conducted in accordance with the Meta-analysis of Observational Studies in Epidemiology and the Preferred Reporting Items for Systematic Reviews and Meta-Analyses statements ${ }^{15}$ (online supplementary file 1). In addition, AMSTAR (A Measurement Tool to Assess Systematic Reviews) was used to assess the methodological quality of this meta-analysis. ${ }^{16} 17$

\section{Search strategy}

Studies in PubMed, Embase, Cochrane Library, Web of Science and China Biology Medicine databases published from the date of inception to 1 December 2018 were systematically searched by TS and QH. The search terms used were as follows: ("Soluble urokinase plasminogen activator receptor" OR "suPAR") AND ("Glomerulosclerosis, Focal Segmental” OR "Segmental Glomerulosclerosis, Focal" OR "Glomerulosclerosis, Focal" OR "Focal Glomerulosclerosis" OR "Sclerosing Glomerulonephritides, Focal" OR "Hyalinosis, Segmental Glomerular") (online supplementary file 2). Selected articles were screened manually to prevent the omission of additional relevant articles. There were no language restrictions. When opinions were not uniform, a third researcher (HX) evaluated and a unified decision was made.

\section{Inclusion and exclusion criteria}

\section{Inclusion criteria}

The following were the inclusion criteria: (1) data on the suPAR level were derived from blood samples; (2) FSGS was diagnosed by biopsy; and (3) randomised controlled trials, cohort studies, case-control studies and cross-sectional studies.

\section{Exclusion criteria}

The following were the exclusion criteria: (1) reviews and case reports; (2) studies on the level of suPAR from urine; and (3) animal studies.

All included studies should involve FSGS and concentration of suPAR. There were no age, gender or region restrictions.

\section{Data extraction and quality assessment}

Data were separately extracted by two authors, and included the author and year of publication, research design, country or region, the aetiology of FSGS, patient characteristics (male and average age percentage), suPAR concentration of primary FSGS, secondary FSGS, MCD, $\mathrm{MN}$ and normal control group, optimal cut-off value, and true positive, true negative, false positive and false negative. Additional discussion was provided when the results were inconsistent.

The Newcastle-Ottawa Scale (NOS) ${ }^{18}$ was used to assess the quality of the cohort studies. The quality of the 21 cohort studies was assessed using NOS, which included three main concepts: selection, comparability and outcome assessment. A score of $\geq 7$ was defined as low risk, a score of 5-7 as medium risk and a score of less than 5 as high risk. The methodological quality of the eight cross-sectional studies included in the current study was assessed by the Agency for Healthcare and Quality (AHRQ) ${ }^{18}$ which consisted of 11 checklists. In all studies, the diagnosis-related study used the Quality Assessment of Diagnostic Accuracy Studies-2, ${ }^{18}$ which contained 11 items that were evaluated as either yes, no or unclear.

\section{Data analysis}

To analyse the data, Stata V.15.0 software was used. Continuous variables were described by the standard mean difference (SMD) and 95\% CI. Heterogeneity was assessed by $\mathrm{I}^{2}$ and $\mathrm{p}$ values. An $\mathrm{I}^{2}$ of $0 \%-50 \%$ was considered as low heterogeneity, $51 \%-75 \%$ was considered as moderate heterogeneity, and more than $75 \%$ was considered as high heterogeneity. When the heterogeneity was under $50 \%$, a fixed-effect model was used. Otherwise, a random-effect model was chosen. ${ }^{19}$ Sensitivity analysis was used when the heterogeneity was more than $50 \%$. Begg's test and Egger's test were used to evaluate publication bias when the included studies contained more than 10 studies. ${ }^{20} \mathrm{P}<0.05$ was considered statistically significant.

For subgroup analysis, in a previous study, no differences were observed between children and adults ${ }^{9}$; however, in another study, it was described that the level of surface suPAR was related to age. ${ }^{21}$ Because the study design may influence the results, we also performed subgroup analysis based on the study design. In one study, ${ }^{9}$ the suPAR level of African-American children was described as different from that of other races. Therefore, we hypothesised that race might influence the study results, and subgroup analysis was performed based on the continent. However, due to the lack of data, subgroup analysis was not performed for the stage of CKD, gender, estimated glomerular filtration rate (eGFR) and the cut-off value of FSGS.

For data processing of the diagnostic part, publication bias was assessed by a Deeks' funnel plot. When $\mathrm{p}>0.05$, no publication bias was considered. We extracted data from the diagnostic $2 \times 2$ table. The effect of the threshold on the diagnostic accuracy of suPAR was evaluated using the Spearman's correlation coefficient between the 


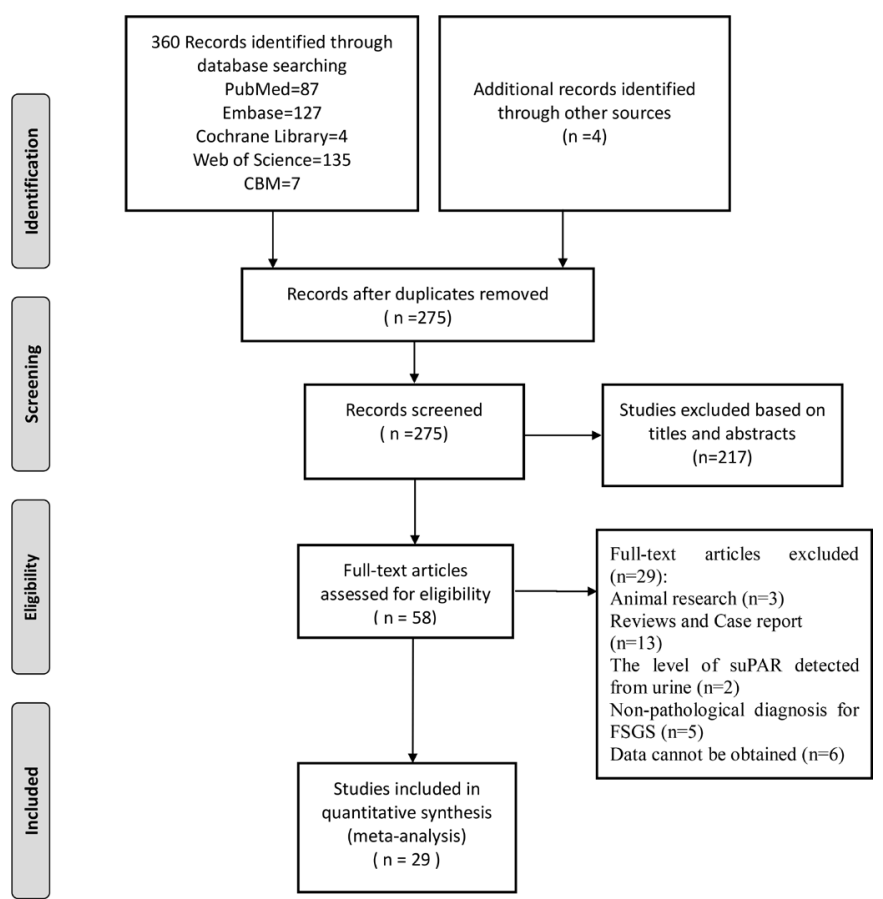

Figure 1 PRISMA (Preferred Reporting Items for Systematic Reviews and Meta-Analyses) flow diagram and exclusion criteria. CBM, China Biology Medicine; FSGS, focal segmental glomerulosclerosis; suPAR, soluble urokinase plasminogen activated receptor.

sensitivity logic and the 1-specific logic. If there was no threshold effect, the mixed sensitivity (SENS), specificity (SPEC), diagnostic OR (DOR), positive likelihood ratio (PLR) and negative likelihood ratio (NLR) were calculated using a bivariate random-effects regression model. In addition, a summary receiver operational characteristic (SROC) curve was created by plotting individual and summary points of sensitivity and specificity to assess overall diagnostic accuracy. Furthermore, the area under the curve (AUC) was obtained, and a forest plot was constructed. Our data showed that the diagnostic value was better when the AUC was closer to 1 .

\section{Patient and public involvement}

This study did not involve patients or members of the public.

\section{RESULTS}

After the initial search, a total of 360 studies were obtained from five databases. Another four studies were included from the sources of reference list. Thus, 364 studies were initially included, among which 306 studies were excluded after reading the title and abstract. After reading the full text, another 29 studies were excluded; therefore, 29 studies were finally included. ${ }^{9-11} 21-46$ A flow chart of the study selection process is presented in figure 1. A total of 5187 patients were involved in the 29 included studies. The characteristics of the included studies are shown in table 1 . Each study included basic information, study types, country, thresholds and quality scores. In seven studies, ${ }^{22} 233031344043$ the cut-off value of suPAR was described, which ranged from 2992.6 to $5500 \mathrm{pg} / \mathrm{mL}$.

\section{Concentration of suPAR in primary FSGS and normal control group}

In total, there were 18 studies $^{9} 10$ 22-27 30 33-35 3738 40-42 46 in which the concentrations of suPAR were compared between primary FSGS and the normal control group. The overall results showed that the level of suPAR in the primary FSGS group was significantly higher when compared with that in the normal control group $(\mathrm{p}<0.001$; SMD: 2.56 ; $95 \%$ CI 1.85 to $\left.3.28 ; \mathrm{I}^{2}=96.9 \%\right)$. Furthermore, the results indicated significant evidence of betweenstudy heterogeneity. Sensitivity analysis was employed, which demonstrated that it did not affect the final results. Therefore, subgroup analysis was performed. In a study by Wei $e t a l{ }^{21}$ there were two cohorts in which the age of the FSGS clinical trial (CT) cohort was mixed (age 0-40 years) and the CodoNet cohort was for children aged 0-18 years old. We named these two cohorts the 'Wei, C.2012-1' and 'Wei, C.2012-1' groups. The results of the subgroup analysis are presented in figure 2. Subgroup analysis according to study design and continent is shown in online supplementary figures 1-2. The funnel plot indicated that there might be publication bias (online supplementary figure 3), and the Begg's test and Egger's test $(\mathrm{p}<0.05)$ showed publication bias.

\section{Secondary FSGS and the normal control group}

In four studies, ${ }^{21} 243637$ the concentrations of suPAR were described between secondary FSGS and the normal control group. In addition, significant differences were observed between the secondary FSGS and the normal control group ( $\mathrm{p}<0.001$; SMD: 1.68 ; $95 \%$ CI 1.37 to 1.98 ; $\mathrm{I}^{2}=0.0 \%$ ) (online supplementary figure 4 ).

\section{Primary FSGS and secondary FSGS}

In a total of four studies, ${ }^{21} 243237$ the concentrations of suPAR in primary FSGS and secondary FSGS were compared. Our data analysis showed that the concentration of suPAR was higher in the secondary FSGS compared with the primary FSGS ( $\mathrm{p}<0.008$; SMD: $0.47 ; 95 \%$ CI -0.07 to $1.01 ; \mathrm{I}^{2}=69.7 \%$ ) (table 2 ).

\section{Primary FSGS and MCD}

In a total of 19 studies, ${ }^{9} 22$ 24-28 3031 33-35 $3738404244-46$ the concentrations of suPAR in primary FSGS and MCD were compared, and the results showed that the concentration of suPAR in primary FSGS was significantly higher compared with that in MCD ( $<<0.001$; SMD: $1.72 ; 95 \%$ CI 1.17 to $2.28 ; \mathrm{I}^{2}=94.0 \%$ ) (table 2 ).

\section{Primary FSGS and MN}

In 16 studies, ${ }^{9} 102224273031333437-4244$ the concentrations of suPAR were compared in primary FSGS and MN, and the results were significantly different $(\mathrm{p}<0.001$; SMD: 0.88 ; $95 \%$ CI 0.50 to $1.27 ; \mathrm{I}^{2}=88.1 \%$ ) (table 2 ). 
은 을 (10)

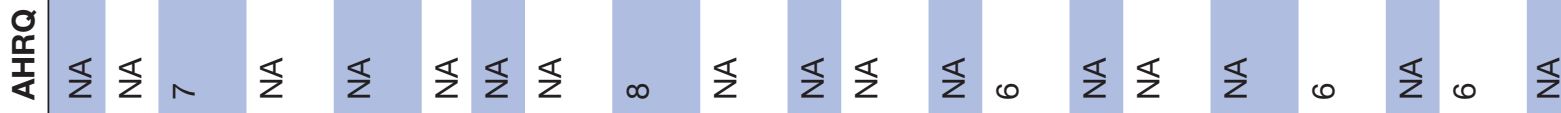

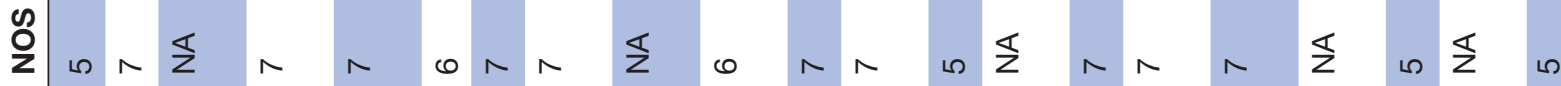

हैं कू

步 $\frac{7}{\frac{\pi}{0}}$

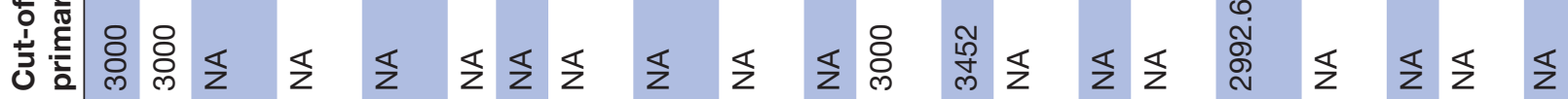

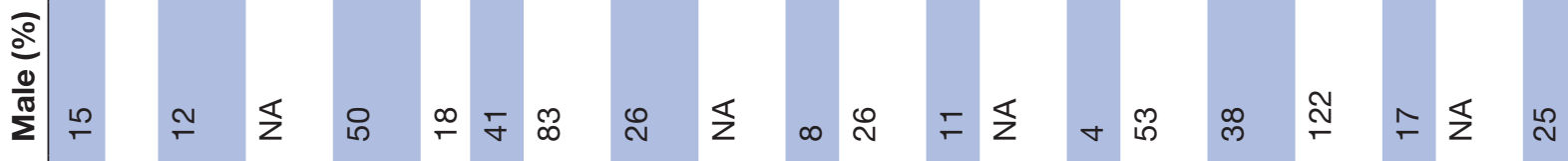

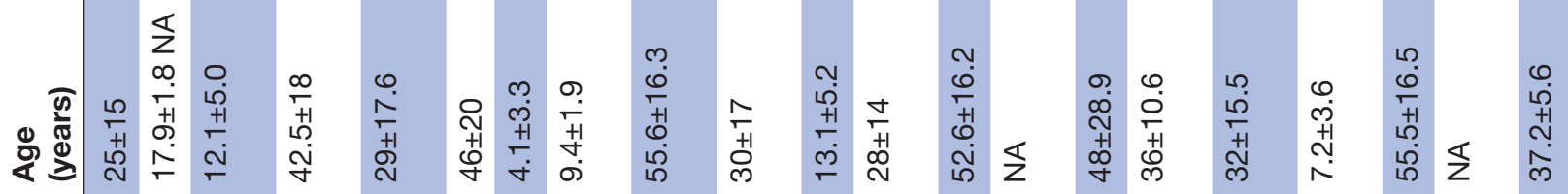

$\frac{0}{\frac{0}{3}}$

高守滎

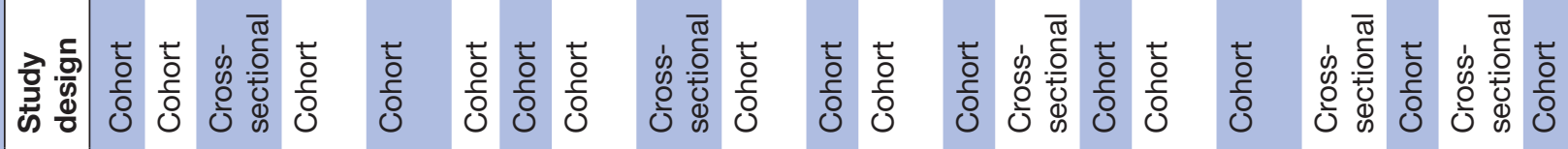

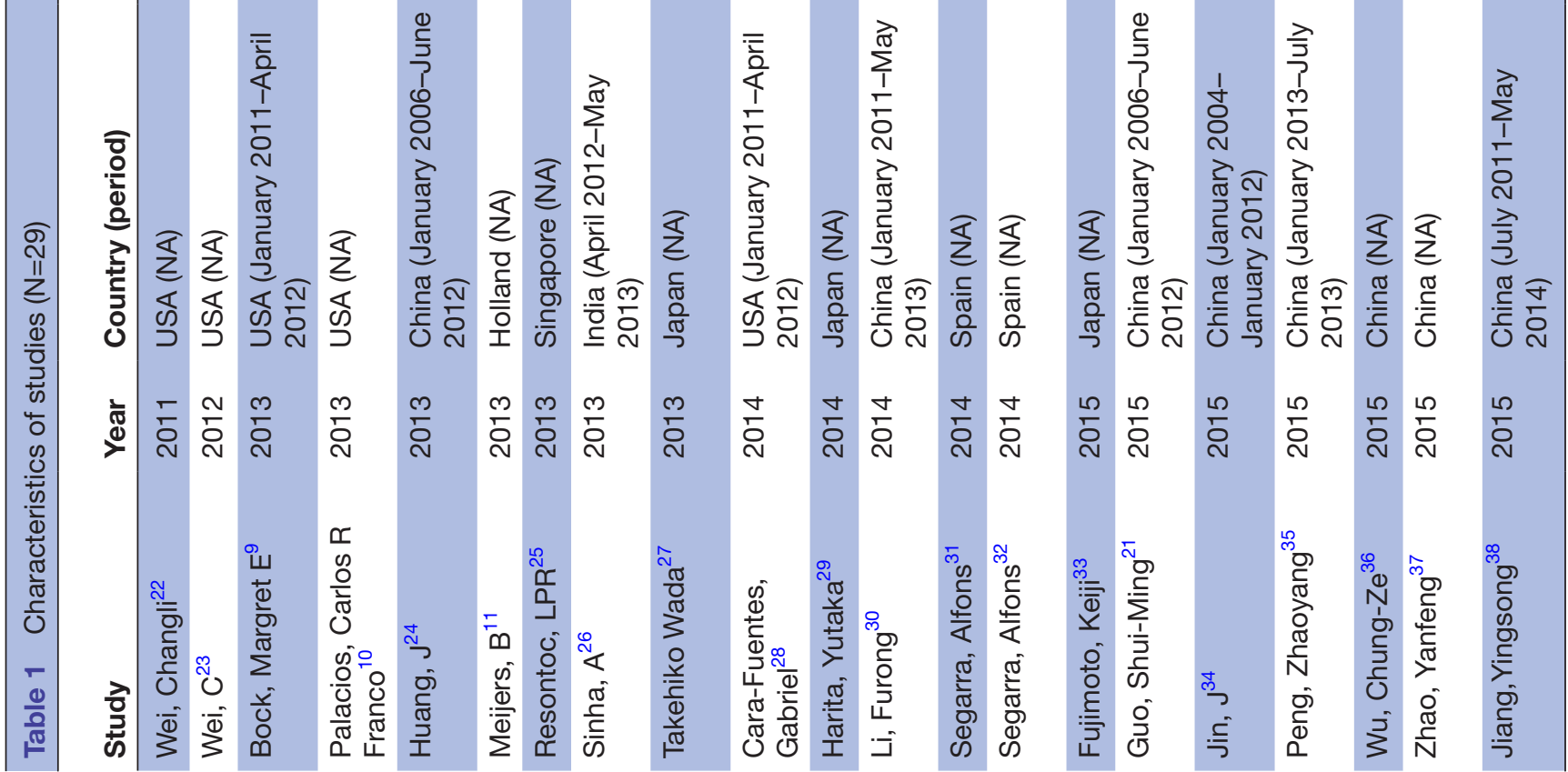




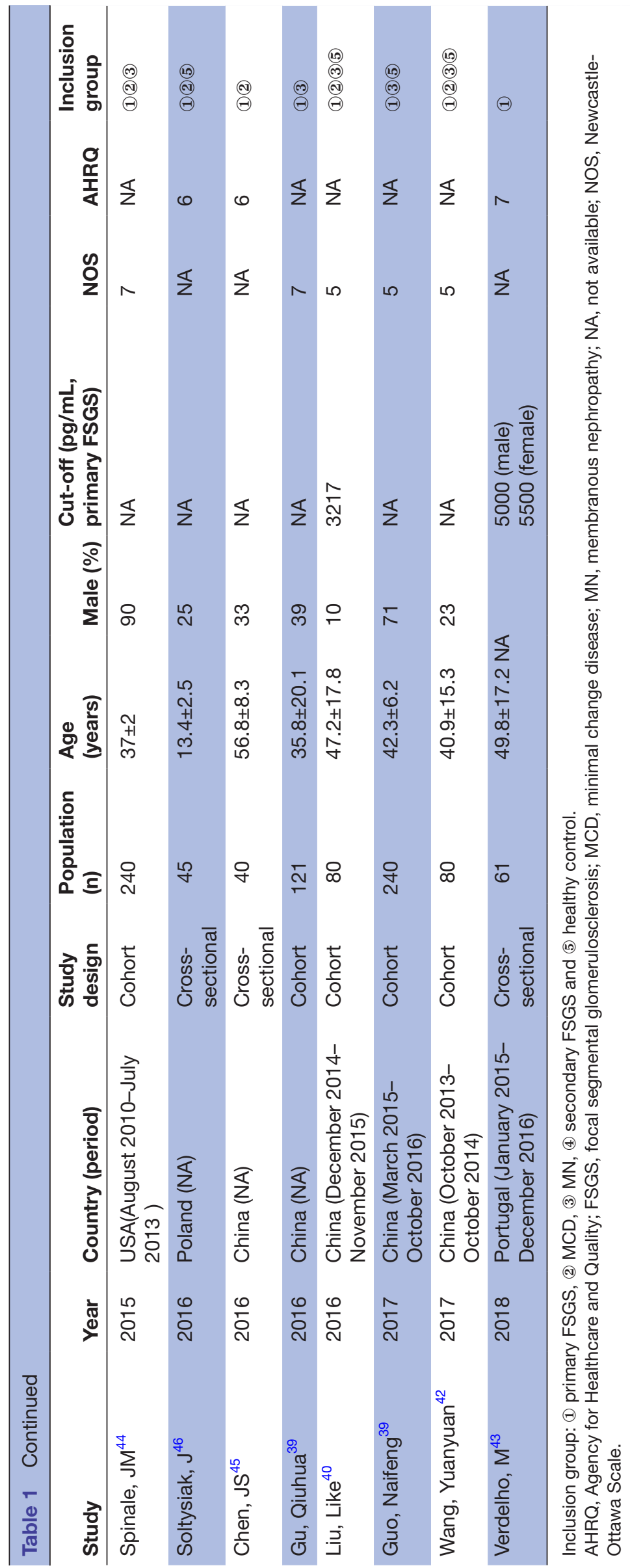




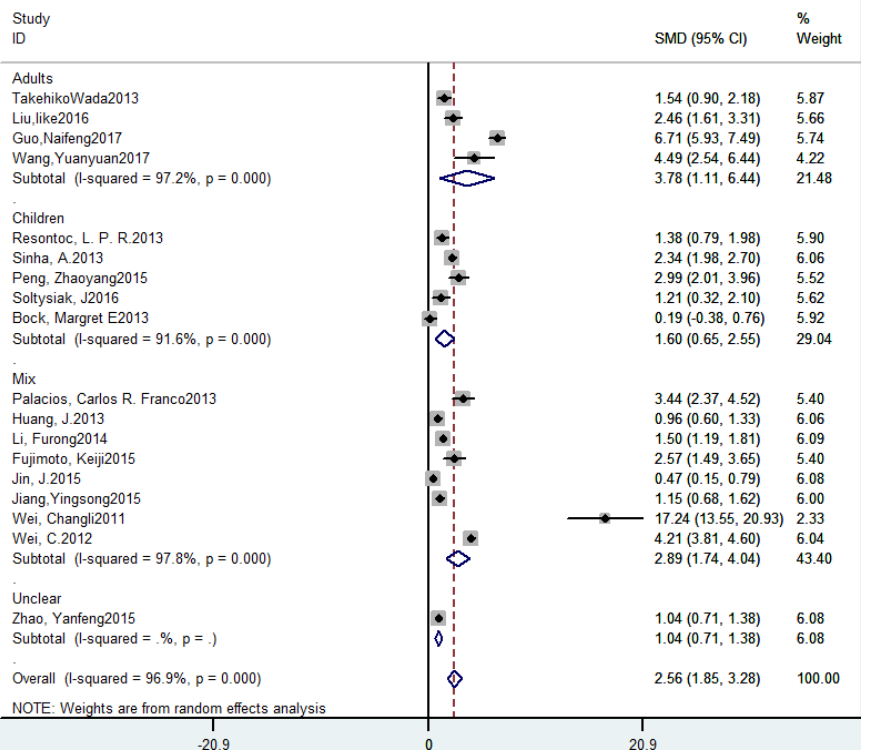

Figure 2 Forest plot for the concentration of SUPAR between FSGS and normal group. FSGS, focal segmental glomerulosclerosis; SMD, standard mean difference; suPAR, soluble urokinase plasminogen activated receptor.

\section{MCD and MN}

In 14 studies, ${ }^{9} 2224273031333436-38404244$ the concentrations of suPAR were compared in MCD and MN, and the results showed that in MCD and MN the concentrations were significantly different ( $\mathrm{p}=0.008$; SMD: $-0.69 ; 95 \%$ CI -1.20 to $0.18 ; \mathrm{I}^{2}=89.8 \%$ ) (table 2 ).

\section{Diagnostic value of suPAR for primary FSGS}

Seven of these studies 22233031344043 involved the threshold of suPAR. We analysed the diagnostic value of suPAR in these studies. suPAR could diagnose primary FSGS (PLR 4.44, $95 \%$ CI 2.21 to 8.95 ; NLR $0.38,95 \%$ CI 0.29 to 0.49 ; DOR $11.86,95 \%$ CI 5.13 to $27.39 ;$ SENS=0.68, $95 \%$ CI 0.59 to 0.76 ; $\mathrm{SPEC}=0.85,95 \% \mathrm{CI} 0.70$ to 0.93 ; SROC curve: AUC $=0.78,95 \%$ CI 0.75 to 0.82 ) (online supplementary figures 5-6).

The results after removing one study ${ }^{43}$ showed higher diagnostic value (PLR 5.94, 95\% CI 3.44 to 10.23; NLR $0.32,95 \%$ CI 0.25 to 0.42 ; DOR $18.34,95 \%$ CI 11.49 to 29.32; $\mathrm{SENS}=0.72,95 \% \mathrm{CI} 0.61$ to $0.80 ; \mathrm{SPEC}=0.88$, $95 \%$ CI 0.78 to 0.94 ; SROC curve: $\mathrm{AUC}=0.85,95 \%$ CI 0.82 to 0.88 ) (figures 3-4). No publication bias was observed

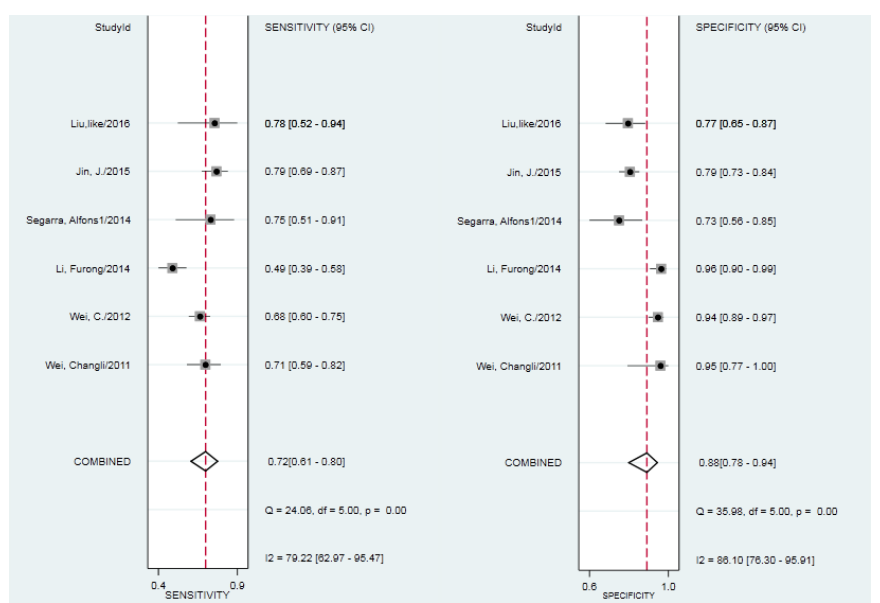

Figure 3 Sensitivity and specificity forest map (the study that described a threshold of $5000 \mathrm{pg} / \mathrm{mL}$ was removed).

in threshold-related studies by Deeks' funnel plot $(\mathrm{p}>0.1)$ (online supplementary figure 7).

\section{Quality assessment}

Of the 21 included cohort studies, ${ }^{10} 11$ 21-26 28-31 $33343638-4244$ the NOS was used to score the quality of the cohort studies. Most studies had a quality score between 5 and 7 and were at moderate risk. In eight cross-sectional studies, ${ }^{927323537434546}$ the AHRQ rating scale was used for scoring, and the scores were between 6 and 8, and were between medium and high quality. In studies 22233031344043 involving the diagnostic part, the QUADS-2 scale was used for quality scoring. All studies were diagnosed using the unified gold standard (pathological biopsy); however, none was performed using blinded conditions (online supplementary tables $1-3)$.

\section{DISCUSSION}

suPAR, a circulating form of the surface receptor in many cells, is a promising biomarker, which is elevated in inflammation, autoimmune diseases, tumours and kidney diseases. ${ }^{6}$ In a previous study, it was demonstrated that suPAR might be causal for kidney disease ${ }^{47}$; however, it was not clear whether suPAR could diagnose kidney disease. Our results showed that suPAR could differentiate primary and secondary FSGS from the normal control group. Our results showed that suPAR could distinguish

\begin{tabular}{lccccc}
\hline \multicolumn{1}{l}{ Table 2} & Results comparing the level of suPAR in different diseases & & \\
\hline Disease & P value & SMD & $\mathbf{9 5 \% ~ C l ~}$ & I $^{\mathbf{2}}$ (\%) & P heterogeneity \\
\hline Primary FSGS vs secondary FSGS & 0.08 & 0.47 & -0.07 to 1.01 & 69.7 & 0.01 \\
Primary FSGS vs MCD & $<0.001$ & 1.72 & 1.27 to 2.28 & 94.0 & $<0.001$ \\
Primary FSGS vs MN & $<0.001$ & 0.88 & 0.50 to 1.27 & 88.1 & $<0.001$ \\
MCD and MN & 0.008 & -0.69 & -1.20 to 0.18 & 89.8 & $<0.001$
\end{tabular}

FSGS, focal segmental glomerulosclerosis; MCD, minimal change disease; MN, membranous nephropathy; SMD, standard mean difference; suPAR, soluble urokinase plasminogen activated receptor. 


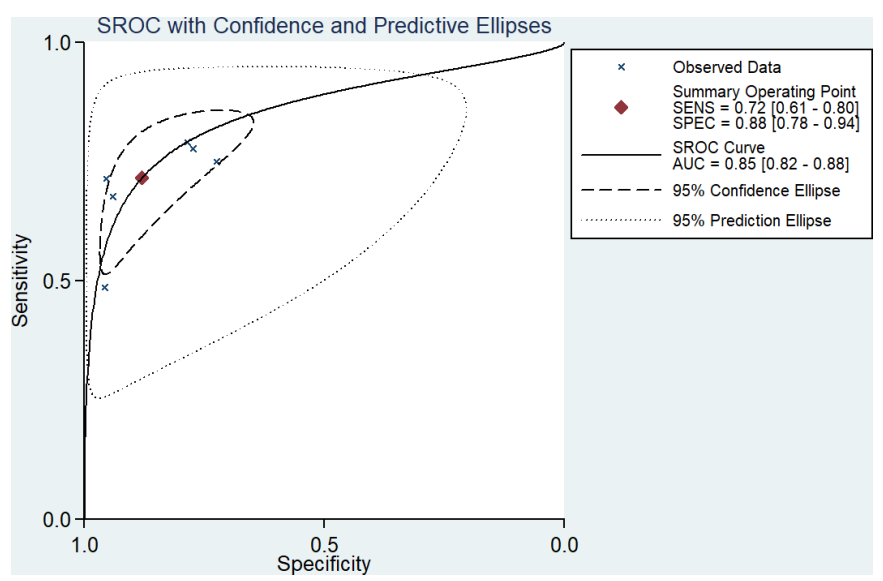

Figure 4 SROC curve for the value of suPAR for FSGS (the study that described a threshold of $5000 \mathrm{pg} / \mathrm{mL}$ was removed). AUC, area under the curve; FSGS, focal segmental glomerulosclerosis; SENS, sensitivity; SPEC, specificity; SROC, summary receiver operational characteristic; suPAR, soluble urokinase plasminogen activated receptor.

primary FSGS and MN and MCD; however, it could not differentiate between primary FSGS and secondary FSGS.

In some studies, ${ }^{8-11}$ it was demonstrated that suPAR could not be used as a biomarker for the diagnosis of primary FSGS; however, other studies $22-25273031$ showed that suPAR could be used as a biomarker for the diagnosis of FSGS. Therefore, the precise diagnostic value of suPAR in FSGS remains unclear. Our results showed that suPAR could diagnose FSGS. We hypothesised that standardisation of measurement techniques for suPAR could be an option. In addition, gender, age and basic kidney function may lead to differences in results. In a letter by Maas et $a l^{48}$ it was demonstrated that the level of suPAR in primary FSGS was not different from that of secondary FSGS, and that there was only minimal change in disease. Despite these results, Huang et $a l^{24}$ showed that when comparing the concentration of suPAR, there was a significant difference between secondary FSGS with haemodynamic diseases and primary FSGS, which was similar to our data. Segarra $e$ t $a l^{31}$ showed that SuPAR levels lack sensitivity to distinguish between idiopathic and secondary FSGS. However, suPAR levels greater than $4000 \mathrm{ng} / \mathrm{mL}$ were highly specific for primary FSGS. suPAR does not differentiate between primary FSGS and secondary FSGS, which may be related to age and kidney function. $^{32}$ In addition, studies have shown different levels of suPAR in different races. ${ }^{9}$

MCD, FSGS and mesangial proliferative glomerulonephritis all belong to idiopathic nephrotic syndrome (INS), which refers to the association of nephritic syndrome and non-specific glomerular abnormalities. ${ }^{49}$ The most common characteristic of pathology in children is MCD and FSGS. ${ }^{50}$ MCD is similar to FSGS in renal pathology at early stages. ${ }^{49}$ Despite the similarity, MCD and FSGS have differences. In FSGS, the number of podocytes decreases, whereas in MCD it remains unchanged. ${ }^{51}$ Therefore, in some studies ${ }^{922446}$ it was attempted to differentiate
MCD from FSGS by suPAR in the early stage. Our results suggested that suPAR may be an early diagnostic factor for FSGS and MCD.

The results of this study demonstrated a suPAR concentration of $3000 \mathrm{pg} / \mathrm{mL}$ could be an early diagnosis of FSGS. Seven studies 22233031344043 have been published involving threshold, and in this study the data of these studies were analysed. The overall results showed that there was a moderate diagnostic value in SuPAR to diagnose FSGS. Because of the extremely high level of suPAR in one study, ${ }^{43}$ the study was removed, which resulted in a much higher diagnostic value in primary FSGS. Therefore, we speculated that a suPAR concentration of $3000 \mathrm{pg} / \mathrm{mL}$ may be an optimal threshold for the diagnosis of FSGS.

Initial results showed that the heterogeneity of primary FSGS and the normal control group was substantial. Therefore, we tried to reanalyse the results using sensitivity analysis, which showed the results remained stable. Consequently, a related subgroup analysis was performed. We analysed the different subgroups of primary FSGS in different continents, different research types, and adults and children, and found that the heterogeneity still existed. In a previous study, the correlation between eGFR and suPAR was analysed ${ }^{52}$; therefore, we considered eGFR as an influencing factor for suPAR concentration. However, due to the lack of relevant data, subgroup analysis was not performed according to eGFR. Steroids were the first-line treatment for FSGS ${ }^{30}$; however, in most included studies, it was not mentioned if steroids were used for treatment. Importantly, in our study, no differences in results were observed between adults and children.

Our results showed that there was publication bias when FSGS was diagnosed by suPAR. We tried to research the database to reduce the publication bias. Eventually, we found that the results of included studies were similar as before, indicating that the results of this study were stable. Moreover, when we tried to diagnose FSGS with the threshold of suPAR concentration, relevant studies did not show publication bias.

\section{Strengths and limitations of this study}

First, we evaluated the effect of increased concentration of suPAR on FSGS, and used sensitivity and specificity analyses to diagnose the threshold of suPAR. This will provide the possibility to perform a blood screen before diagnosing FSGS, and based on the results patients with high suPAR concentrations may undergo renal biopsy. In addition, elderly patients or patients who refuse to undergo invasive examination may be predicted by blood tests. Second, we also analysed whether suPAR could distinguish INS (MCD, MN and FSGS), which may help us treat primary kidney disease by the cause of the disease. Third, we used three scales for different articles to evaluate their quality.

Our meta-analysis has some limitations. First, there is publication bias and heterogeneity in part of the results 
of our study. We tried to identify the origin of bias and heterogeneity by sensitivity and subgroup analyses. Considering the many factors that affect heterogeneity, we used age, study design, continent, eGFR and gender subgroup for analysis. Ultimately, we conducted subgroup analysis of age, study design and continent. Since data on pathogeny and gender were not available, no subgroup analysis of these groups was performed. Second, many diseases affect plasma suPAR levels, including tumours, infections, atherosclerosis and autoimmune diseases, and different measurement methods may interfere with the results. Finally, a small percentage of the data were obtained through the reading software, which may have affected the accuracy of the data.

\section{CONCLUSION}

In conclusion, this meta-analysis shows that serum suPAR levels are a potential biomarker for the diagnosis of FSGS. However, considering publication bias, heterogeneity and sample size, additional studies will be required to verify the data.

\section{Author affiliations}

${ }^{1}$ Department of Intensive Care Unit, Lanzhou University First Affiliated Hospital, Lanzhou, China

${ }^{2}$ Evidence-Based Medicine Center, School of Basic Medical Sciences, Lanzhou University, Lanzhou, China

${ }^{3}$ The First Clinical Medical College of the First Hospital of Lanzhou University, Lanzhou, China

${ }^{4}$ Institute of Clinical Research and Evidence Based Medicine, Gansu Province People's Hospital, Lanzhou, China

${ }^{5}$ Evidence Based Social Science Research Center, Lanzhou University, Lanzhou, China

${ }^{6}$ Institute of Evidence Based Rehabilitation Medicine of Gansu Province, Lanzhou University, Lanzhou, China

${ }^{7}$ Key Laboratory of Evidence Based Medicine and Knowledge Translation of Gansu Province, Lanzhou, China

Acknowledgements The authors gratefully acknowledge the support of the First Clinical Hospital of Lanzhou University (Lanzhou, China), the First Clinical Medical College of Lanzhou University, Evidence Based Medicine Center of Lanzhou University, and all the authors who participated in this study.

Contributors TS and PY designed the experiments. TS, QH and HX searched articles in the database. TS, JL, JinL and LZ wrote the manuscript. JL and KY checked the manuscript. All authors reviewed and agreed with the content of the manuscript.

Funding This study was supported by the Laboratory of Intelligent Medical Engineering of Gansu Province (GSXZYZH2018001). The authors remain independent of any funding influence.

Competing interests None declared.

Patient consent for publication Not required.

Provenance and peer review Not commissioned; externally peer reviewed.

Open access This is an open access article distributed in accordance with the Creative Commons Attribution Non Commercial (CC BY-NC 4.0) license, which permits others to distribute, remix, adapt, build upon this work non-commercially, and license their derivative works on different terms, provided the original work is properly cited, appropriate credit is given, any changes made indicated, and the use is non-commercial. See: http://creativecommons.org/licenses/by-nc/4.0/.

\section{ORCID iD}

Liu Jian http://orcid.org/0000-0002-1825-571X

\section{REFERENCES}

1. Peev V, Hahm E, Reiser J. Unwinding focal segmental glomerulosclerosis. F1000Res 2017;6.

2. Hill NR, Fatoba ST, Oke JL, et al. Global Prevalence of Chronic Kidney Disease - A Systematic Review and Meta-Analysis. PLoS One 2016;11:e0158765

3. Roth GA, Abate D, Abate $\mathrm{KH}$, et al. Global, regional, and national age-sex-specific mortality for 282 causes of death in 195 countries and territories, 1980-2017: a systematic analysis for the global burden of disease study 2017. Lancet 2018;392:1736-88.

4. Rosenberg AZ, Kopp JB. Focal segmental glomerulosclerosis. Clin J Am Soc Nephrol 2017;12:502-17.

5. Bunch DR, El-Khoury JM. Emerging biomarker of kidney disease: suPAR or Subpar? Clin Chem 2018;64:1545-7.

6. Hamie L, Daoud G, Nemer G, et al. SuPAR, an emerging biomarker in kidney and inflammatory diseases. Postgrad Med J 2018;94:517-24.

7. Hall SS. Omen in the blood. Science 2018;360:254-8.

8. Skorecki KL, Freedman BI. A suPAR biomarker for chronic kidney disease. N Engl J Med 2015;373:1971-2.

9. Bock ME, Price HE, Gallon L, et al. Serum soluble urokinasetype plasminogen activator receptor levels and idiopathic FSGS in children: a single-center report. Clin J Am Soc Nephrol 2013;8:1304-11.

10. Franco Palacios CR, Lieske JC, Wadei HM, et al. Urine but not serum soluble urokinase receptor (suPAR) may identify cases of recurrent FSGS in kidney transplant candidates. Transplantation 2013;96:394-9.

11. Meijers B, Maas RJH, Sprangers B, et al. The soluble urokinase receptor is not a clinical marker for focal segmenta glomerulosclerosis. Kidney Int 2014;85:636-40.

12. Yao L, Sun R, Chen Y-L, et al. The quality of evidence in Chinese meta-analyses needs to be improved. J Clin Epidemiol 2016;74:73-9.

13. Tian J, Zhang J, Ge L, et al. The methodological and reporting quality of systematic reviews from China and the USA are similar. $J$ Clin Epidemiol 2017;85:50-8.

14. Lee JM, Yang JW, Kronbichler A, et al. Increased serum soluble urokinase-type plasminogen activator receptor (suPAR) levels in FSGS: a meta-analysis. Journal of Immunology Research 2019;2019:1-11.

15. Panic N, Leoncini E, de Belvis G, et al. Evaluation of the endorsement of the preferred reporting items for systematic reviews and meta-analysis (PRISMA) statement on the quality of published systematic review and meta-analyses. PLoS One 2013;8:e83138.

16. Pieper D, Buechter RB, Li L, et al. Systematic review found AMSTAR, but not R(evised)-AMSTAR, to have good measurement properties. J Clin Epidemiol 2015;68:574-83.

17. Yan $\mathrm{P}, \mathrm{Yao} \mathrm{L}$, Li H, et al. The methodological quality of robotic surgical meta-analyses needed to be improved: a cross-sectional study. J Clin Epidemiol 2019;109:20-9.

18. Zeng $X$, Zhang $Y$, Kwong JSW, et al. The methodological quality assessment tools for preclinical and clinical studies, systematic review and meta-analysis, and clinical practice guideline: a systematic review. J Evid Based Med 2015;8:2-10.

19. Higgins JPT, Green S, eds. Cochrane handbook for systematic reviews of interventions 4.2.5 [updated May 2005]. Chichester, UK: The Cochrane Library, John Wiley \& Sons, Ltd, 2005.

20. Song F, Gilbody S. Bias in meta-analysis detected by a simple, graphical test. increase in studies of publication bias coincided with increasing use of meta-analysis. BMJ 1998;316:471.

21. Guo S-M, Han M, Chen M-X, et al. Soluble urokinase receptor levels are correlated with focal segmental glomerulosclerosis lesions in IgA nephropathy: a cohort study from China. PLoS One 2015;10:e0138718.

22. Wei C, El Hindi S, Li J, et al. Circulating urokinase receptor as a cause of focal segmental glomerulosclerosis. Nat Med 2011;17:952-60.

23. Wei C, Trachtman $\mathrm{H}$, Li J, et al. Circulating suPAR in two cohorts of primary FSGS. J Am Soc Nephrol 2012;23:2051-9.

24. Huang J, Liu G, Zhang Y-M, et al. Plasma soluble urokinase receptor levels are increased but do not distinguish primary from secondary focal segmental glomerulosclerosis. Kidney Int 2013;84:366-72.

25. Resontoc LPR, Chan CY, Liang AW, et al. Circulating soluble urokinase-type plasminogen activator receptor (SUPAR) does not predict treatment outcomes inmulti-ethnicasian children with idiopathic focal segmental glomerulosclerosis (FSGS). Pediatr Nephrol 2013;28.

26. Sinha A, Bajpai J, Saini S, et al. Serum-Soluble urokinase receptor levels do not distinguish focal segmental glomerulosclerosis from other causes of nephrotic syndrome in children. Kidney Int 2014;85:649-58. 
27. Wada T, Nangaku M, Maruyama S, et al. A multicenter crosssectional study of circulating soluble urokinase receptor in Japanese patients with glomerular disease. Kidney Int 2014;85:641-8.

28. Cara-Fuentes G, Wei C, Segarra A, et al. Cd80 and suPAR in patients with minimal change disease and focal segmental glomerulosclerosis: diagnostic and pathogenic significance. Pediatr Nephrol 2014;29:1363-71.

29. Harita Y, Ishizuka K, Tanego A, et al. Decreased glomerular filtration as the primary factor of elevated circulating suPAR levels in focal segmental glomerulosclerosis. Pediatr Nephrol 2014;29:1553-60.

30. Li F, Zheng C, Zhong Y, et al. Relationship between serum soluble urokinase plasminogen activator receptor level and steroid responsiveness in FSGS. Clin J Am Soc Nephrol 2014;9:1903-11.

31. Segarra A, Jatem E, Quiles MT, et al. [Diagnostic value of soluble urokinase-type plasminogen activator receptor serum levels in adults with idiopathic nephrotic syndrome]. Nefrologia 2014;34:46-52.

32. Segarra A, Jatem E, Quiles MT, et al. [Value of soluble urokinase receptor serum levels in the differential diagnosis between idiopathic and secondary focal segmental glomerulosclerosis]. Nefrologia 2014;34:53-61.

33. Fujimoto K, Imura J, Atsumi H, et al. Clinical significance of serum and urinary soluble urokinase receptor (suPAR) in primary nephrotic syndrome and MPO-ANCA-associated glomerulonephritis in Japanese. Clin Exp Nephrol 2015;19:804-14.

34. Jin J, Li YW, He Q. Primary and recurrent focal segmental glomerulosclerosis closely link to serum soluble urokinasetype plasminogen activator receptor levels. Transplant Proc 2015;47:1760-5

35. Peng Z, Mao J, Chen X, et al. Serum suPAR levels help differentiate steroid resistance from steroid-sensitive nephrotic syndrome in children. Pediatric Nephrology 2015;30:301-7.

36. Wu C-Z, Chang L-C, Lin Y-F, et al. Urokinase plasminogen activator receptor and its soluble form in common biopsy-proven kidney diseases and in staging of diabetic nephropathy. Clin Biochem 2015;48:1324-9.

37. Zhao Y, Liu L, Huang J, et al. Plasma soluble urokinase receptor level is correlated with podocytes damage in patients with $\lg A$ nephropathy. PLoS One 2015;10:e0132869.

38. Yingsong J, Song L, Wei L. Expression and clinical significance of soluble urokinase-type plasminogen activator receptor in serum of patients with focal segmental glomerulosclerosis. Chongqing Medical 2015;44:2829-31.

39. Gu Q-H, Cui Z, Huang J, et al. Patients with combined membranous nephropathy and focal segmental glomerulosclerosis have comparable clinical and autoantibody profiles with primary membranous nephropathy: a retrospective observational study. Medicine (Baltimore) 2016;95:e3786.

40. Like L. Expression of soluble urokinase-type plasminogen activator receptor in serum of adult patients with primary nephrotic syndrome and its clinical significance. Hebei Medical University 2016;04:1-48

41. Naifeng G, Yingjie C, Li Y, et al. Changes of serum Angptl 4 and suPAR in patients with MsPGN and FSGS and their clinical significance. J Med Internet Res 2017;46:140-3.

42. Yuanyuan W, Linchun D, Qiuling F, et al. Levels and significance of serum soluble urokinase-type plasminogen activator receptor in patients with different pathological types of adult nephrotic syndrome. Chinese Journal of Integrated Traditional and Western Nephrology 2017;18:687-91.

43. Verdelho M, Carina Ferreira A, Céu Santos M, et al. Soluble urokinase-type plasminogen activator receptor as a biomarker for focal segmental glomerulosclerosis; a retrospective analysis. $J$ Nephropathol 2018;7:182-7.

44. Spinale JM, Mariani LH, Kapoor S, et al. A reassessment of soluble urokinase-type plasminogen activator receptor in glomerular disease. Kidney Int 2015;87:564-74.

45. Chen J-S, Chang L-C, Wu C-Z, et al. Significance of the urokinasetype plasminogen activator and its receptor in the progression of focal segmental glomerulosclerosis in clinical and mouse models. $J$ Biomed Sci 2016;23:24.

46. Soltysiak J, Zachwieja J, Benedyk A, et al. Ostalska-Nowicka D: circulating suPAR as a biomarker of disease severity in children with proteinuric glomerulonephritis. Minerva Pediatr 2019;71:4-11.

47. Hayek SS, Koh KH, Grams ME, et al. A tripartite complex of suPAR, APOL1 risk variants and $\alpha \beta_{3}$ integrin on podocytes mediates chronic kidney disease. Nat Med 2017;23:945-53.

48. Maas RJH, Wetzels JFM, Deegens JKJ. Serum-Soluble urokinase receptor concentration in primary FSGS. Kidney Int 2012;81:1043-4.

49. Barisoni L, Schnaper HW, Kopp JB. A proposed taxonomy for the podocytopathies: a reassessment of the primary nephrotic diseases. Clin J Am Soc Nephrol 2007;2:529-42.

50. Noone DG, lijima K, Parekh R. Idiopathic nephrotic syndrome in children. Lancet 2018;392:61-74.

51. Kriz W, LeHir M. Pathways to nephron loss starting from glomerular diseases-insights from animal models. Kidney Int 2005;67:404-19.

52. Kronbichler A, Saleem MA, Meijers B, et al. Soluble urokinase receptors in focal segmental glomerulosclerosis: a review on the scientific point of view. J Immunol Res 2016;2016:1-14 